POS $\quad$ PROCEEDINGS

\title{
The European Pulsar Timing Array
}

Mark Purver, ${ }^{* \dagger}$ Christine Jordan, Michael Kramer, Andrew Lyne, Ben Stappers

Jodrell Bank Centre for Astrophysics, University of Manchester, UK

E-mail: mark.purver@postgrad.manchester.ac.uk

\section{Axel Jessner, Kosmas Lazaridis}

Max-Planck-Institut für Radioastronomie, Bonn, Germany

\section{Gemma Janssen, Ramesh Karuppusamy}

Astronomical Institute Anton Pannekoek, University of Amsterdam, Netherlands

\section{Ismaël Cognard, Gregory Desvignes, Gilles Theureau}

Laboratoire de Physique et Chimie de l'Environnement, CNRS, Orléans, France

\section{Marta Burgay, Alessandro Corongiu, Nicolo D'Amico, Andrea Possenti}

INAF - Osservatorio Astronomica di Cagliari, Capoterra, Italy

The European Pulsar Timing Array (EPTA) is a collaboration between the five largest radio telescopes in Europe: Effelsberg in Germany, Westerbork in the Netherlands, Nançay in France, the Lovell Telescope at Jodrell Bank in the UK and the Sardinia Radio Telescope in Italy, which is under construction. These facilities will combine their observations of an array of millisecond pulsars, with the primary aim of detecting a stochastic gravitational wave background at the Earth. For maximum sensitivity, the EPTA will also collaborate with other timing projects to produce a global timing array. In the future, the Square Kilometre Array (SKA) is very likely to be used to study gravitational waves using pulsars.

From Planets to Dark Energy: the Modern Radio Universe

October 1-5 2007

The University of Manchester, $U K$

\footnotetext{
* Speaker.

$\dagger$ This author's work is supported by a grant from the Science and Technology Facilities Council (STFC).
} 


\section{Gravitational Wave Detection with Pulsar Timing Arrays (PTAs)}

Timing of the regular radio pulses received from a pulsar as it rotates can be used in isolation to derive its rotational, astrometric and orbital parameters. This essentially involves fitting for patterns in a time series of residuals, which are the differences between modelled and observed pulse arrival times (times of arrival or TOAs). Fast-spinning millisecond pulsars are generally the most regular rotators, making them the most suitable for accurate timing of other parameters.

TOAs from multiple pulsars can also be analysed together to allow highly sensitive detection of physical effects that alter the path lengths of pulsar signals in a correlated way. One such effect is a stochastic background of gravitational waves, which is believed to permeate the Universe isotropically and represents the sum of the weak, propagating distortions of space emanating from non-spherically symmetric accelerations of mass such as coalescing black hole binary systems and processes in the early Universe (Figure 1(a)). The background at Earth would produce a distinctive signature in the correlated residuals of a PTA according to general relativity: the time-averaged product of the residuals of each pair of pulsars plotted against their angular separation would yield a correlation function like that in Figure 1(b).

A PTA would be sensitive to gravitational waves of nanohertz frequencies, complementing present and future interferometer searches in the millihertz to kilohertz range, such as LIGO and LISA [2]. Estimates of the black hole merger rate suggest that a positive detection is possible with regular timing of 20 pulsars at $\sim 100-500 \mathrm{~ns}$ accuracy per residual for five years [4] [8]. This level of precision can be achieved with current instruments [7].
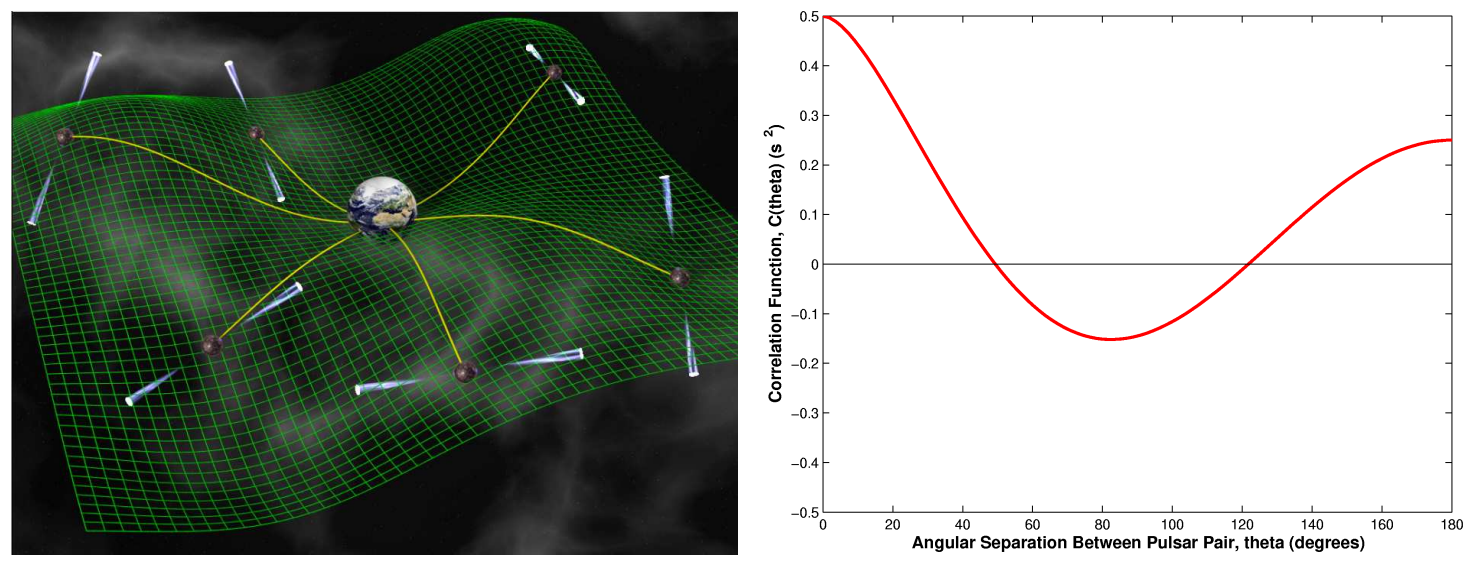

Figure 1: (a) Pulsar signals perturbed by gravitational waves (David Champion).

(b) An idealised pulsar correlation function due to a stochastic gravitational wave background.

\section{A PTA with Multiple Telescopes}

Combination of the five facilities within the EPTA offers a dense time sampling of TOAs, as well as internal consistency checks to correct for systematic deviations between individual telescopes. Additionally, it is possible to conduct regular timing at a range of frequencies. This is necessary in order to keep track of fluctuations in each pulsar's dispersion measure (DM), a frequency-dependent signal delay caused by the ionised interstellar medium. DM corrections can 
be required every few days or weeks to achieve the timing accuracy required for gravitational wave detection [8].

To maximise consistency between telescopes, the EPTA will eventually use common synthetic pulse templates, where each template will be a noise-free fit to a pulsar amplitude profile. In the long term, the institutions aim to develop a common hardware backend for pulsar data processing.

\section{Recent Work}

EPTA residuals have been combined for several millisecond pulsars in order to resolve systematic differences, reduce RMS random error and assess their intrinsic amenability to high-precision timing. Around 12 years of data from Effelsberg, Westerbork, Nançay and Jodrell Bank for the binary pulsar J1518+4904 yielded a residual RMS of $5.2 \mu \mathrm{s}$. This pulsar has a rotational period of $40.9 \mathrm{~ms}$ and an orbital period of 8.63 days. The residuals were used to constrain system parameters [3], such as the relativistic rate of orbital periastron advance to $0.011372(4) \mathrm{deg}_{\mathrm{yr}^{-1}}$, the total

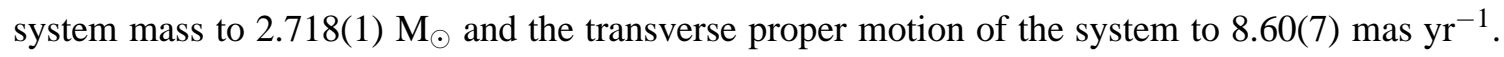
A synthetic template consisting of a sum of four Gaussian components, and a high signal-to-noise template representing a total integration time of 79.6 hours, are shown in Figures 2(a) and 2(b).
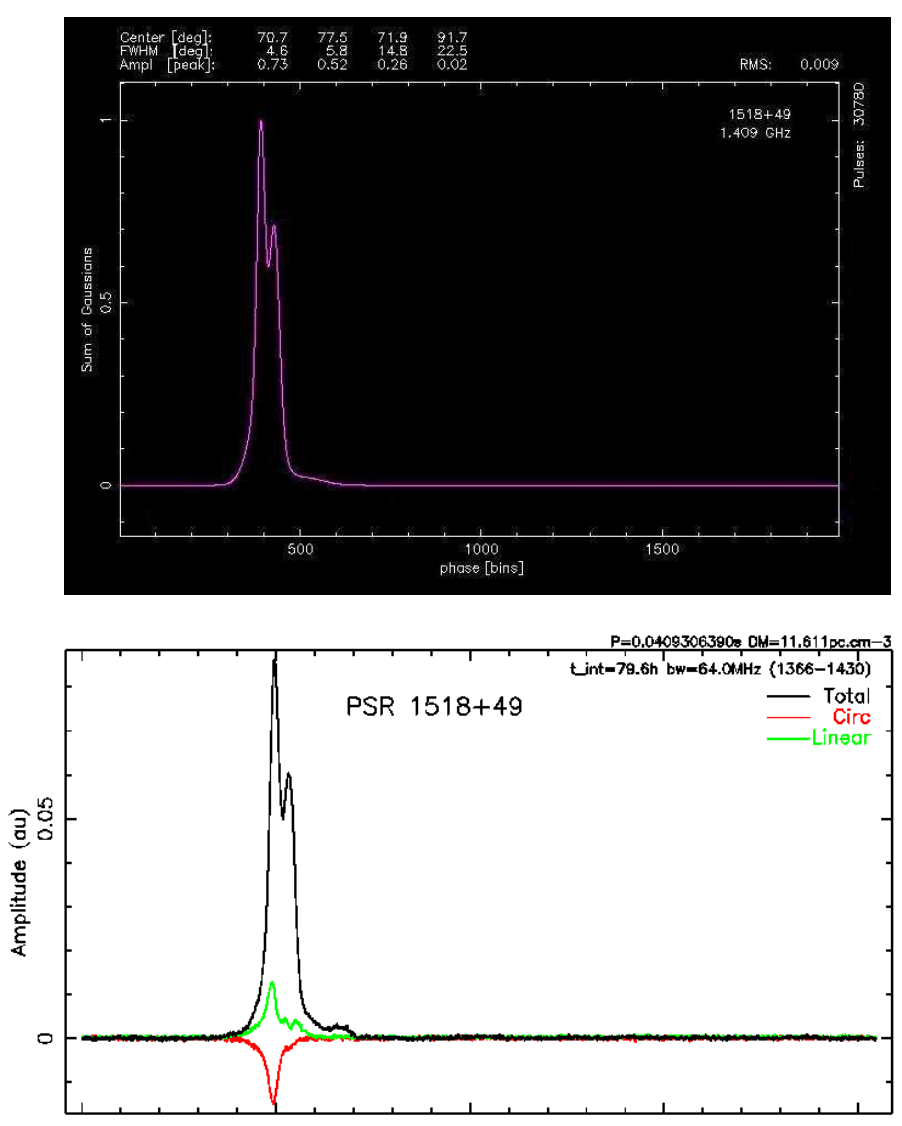

Figure 2: (a) A synthetic template for the total intensity of PSR J1518+4903.

(b) A high signal-to noise template for PSR J1518+4903: total intensity in black; linearly polarised component in green; circularly polarised component in red. 


\section{The Future: Global Collaboration and the SKA}

To maximise the likelihood of gravitational wave detection, a PTA studying all known suitable pulsars is desirable. To this end, the EPTA will collaborate with the Parkes Pulsar Timing Array (PPTA) in Australia [6] and the first PTA, developed in the USA [5], to correlate residuals over the greatest possible number of pulsars. Both these PTAs have a stated aim to detect gravitational waves. From next year, Effelsberg, Jodrell Bank and Sardinia will employ ATNF digital filterbanks of the type used at the Parkes Telescope to process pulsar data, aiding comparability between their residuals.

The arrival of the Square Kilometre Array (SKA), around 2020, will bring a much greater level of accuracy to pulsar timing and uncover many presently undiscovered millisecond pulsars, almost certainly allowing gravitational wave analysis through timing residuals. Timing features in one of the SKA's five key science projects [1].

\section{References}

[1] C. L. Carilli and S. Rawlings, Motivation, key science projects, standards and assumptions, New Astronomy Reviews 48 (2004) 979.

[2] J. Hough and S. Rowan, Gravitational Wave Detection by Interferometry (Ground and Space), Living Rev. Relativity 3 (2000) 3.

[3] G. H. Janssen, B. W. Stappers, M. Kramer, A. Jessner, I. Cognard, D. J. Nice and M. B. Purver, EPTA Timing of PSR J1518+4904, in prep (2007).

[4] F. A. Jenet, G. B. Hobbs, K. J. Lee and R. N. Manchester, Detecting the Stochastic Gravitational Wave Background Using Pulsar Timing, ApJ 625 (2005) L123.

[5] A. N. Lommen and D. C. Backer, New Limits on the Gravitational Wave Background and Other Results from the Pulsar Timing Array, Bull. Amer. Astr. Soc. 33 (2001) 1347.

[6] R. N. Manchester, The Parkes Pulsar Timing Array, Chinese J. Astron. Astrophys. 6 (2006) 139.

[7] W. van Straten, High-Precision Timing and Polarimetry of PSR J0437-4715, PhD Thesis (2003).

[8] X. P. You and 13 co-authors, Dispersion measure variations and their effect on precision pulsar timing, MNRAS 378 (2007) 493. 